\title{
Probing Electronic Fluxes via Time-Resolved X-ray Scattering
}

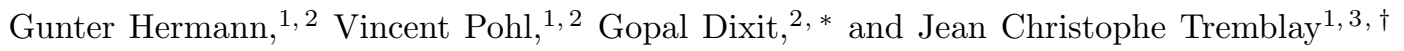 \\ ${ }^{1}$ Institut für Chemie und Biochemie, Freie Universität Berlin, Takustraße 3, D-14195 Berlin, Germany \\ ${ }^{2}$ Department of Physics, Indian Institute of Technology Bombay, Powai, Mumbai 400076 India \\ ${ }^{3}$ Laboratoire de Physique et Chimie Théoriques, CNRS-Université de Lorraine, \\ UMR 7019, ICPM, 1 Bd Arago, 57070 Metz, France
}

(Dated: February 4, 2020)

\begin{abstract}
The current flux density is a vector field that can be used to describe theoretically how electrons flow in a system out-of-equilibrium. In this work, we unequivocally demonstrate that the signal obtained from time-resolved X-ray scattering does not only map the time-evolution of the electronic charge distribution, but also encodes information about the associated electronic current flux density. We show how the electronic current flux density qualitatively maps the distribution of electronic momenta and reveals the underlying mechanism of ultrafast charge migration processes, while also providing quantitative information about the timescales of electronic coherences.
\end{abstract}

Time-resolved imaging of dynamically evolving electronic charge distribution is essential for complete understanding of complex chemical and biological processes in nature. Imaging of valence electron charge distribution is paramount to understand different instances during chemical reactions such as conformational changes, charge migration, and bond formation and breakage [14. Following the quantum continuity equation, the flow of electron is accompanied by associated fluxes [5]. The latter offers a wealth of information and has played a decisive role for understanding chemical reaction mechanisms [6 19. However, the notion of electronic fluxes has been restricted to theoretical modelling [17+26 and there is no general way to probe them directly in experiment. In this work, we demonstrate theoretically real-space and real-time imaging of electronic fluxes associated with charge migration using time-resolved X-ray scattering (TRXS). For this purpose, we consider oriented benzene as a test system in which a pump pulse induces adiabatic charge migration and ultrashort X-ray pulses probe the electronic fluxes accompanying charge migration.

Scattering of X-rays from matter is an invaluable technique to unveil the real-space structure of solids and molecules with atomic-scale resolution 27. Tremendous technological progress has been made to generate tunable ultraintense and ultrashort pulses from X-ray freeelectron lasers (XFELs) 28 30]. X-ray pulses with few femtoseconds pulse duration are routinely generated at various XFELs (LCLS, SACLA, European XFEL). Moreover, few successful attempts have been demonstrated to generate attosecond X-ray pulses [31 36]. The availability of these ultrashort X-ray pulses offer to extend X-ray scattering from static to time domain with unprecedented temporal resolution [37, 38. Scattering of ultrashort Xray pulses from the evolving electronic charge distribution promises to provide stroboscopic snapshots of matter in action with atomic-scale spatial and temporal resolutions [39, 40]. A direct approach to envision TRXS is a pump-probe experiment, where the pump pulse trig- gers the ultrafast dynamics and the induced dynamics is imaged by the ultrashort X-ray pulses. Not only these ultrashort X-ray pulses allow to map the motion of atoms in matter on the femtosecond timescale [40, 41, but also to record movies of electronic motion taking place from few femtoseconds to the attosecond timescale [42, 43].

The availability of ultrashort X-ray pulses has prompted TRXS experiments probing ultrafast processes with atomic-scale spatio-temporal resolutions. Static Xray scattering from aligned 2,5-diiodobenzonitrile has been performed at LCLS 44]. TRXS experiments allowed imaging ultrafast vibrations in iodine [45. Frequency-resolved TRXS was used to disentangle bound and dissociative electronic states during ultrafast vibrational dynamics in iodine 46. Photoinduced structural change during ring opening electrocyclic chemical reaction in cyclohexadiene [47, 48, and cis-trans photochemical structural changes in photoactive yellow protein [49] were captured by TRXS. Anisotropic TRXS measurements have been used to determine transition dipole moment and assign excited electronics states in molecule [50]. Different formalisms have been developed to simulate TRXS from non-equilibrium states of matter [16, 42, 51 59. It was demonstrated that the scattering signal obtained via TRXS from an electronic wavepacket is not associated with the Fourier transform of instantaneous electron density 52, 51, 52, 54, 58. Mukamel and co-workers have proposed that TRXS is capable to probe molecular nonadiabatic dynamics at avoided crossings and conical intersections [60, 61] Also, frequency- and wavevector-resolved TRXS has been used to probe the electron dynamics in molecules [58. Recently, it was shown that TRXS can probe electronic coherences among electronic states 62, and that TRXS from diatomic molecules are not centrosymmetric 63 .

The main focus of this work is to illustrate the capability of TRXS for imaging quantum fluxes during non-stationary charge migration in a coherent electronic wavepacket prepared by an ultrashort pump pulse. Quantum fluxes find their origin in the interferences 
among quantum mechanical phases. The time-resolved response signal that can be extracted from a TRXS experiment contains information about these electronic coherences, and it is therefore suitable for mapping the current flux density. By following the time-evolution of the coherent electronic wavepacket, we demonstrate the relation between the quantum continuity equation for nonstationary charge migration and TRXS.

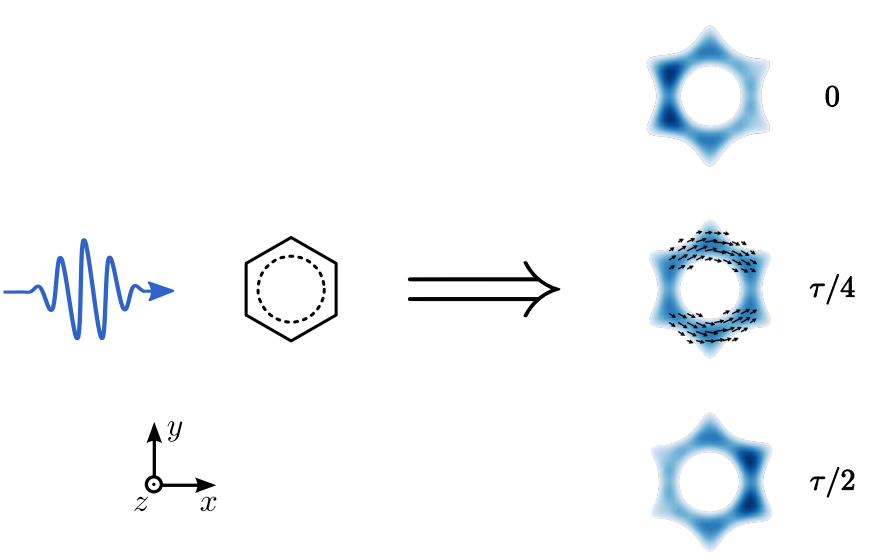

FIG. 1. Conceptual sketch of the charge migration mechanism. An $x$-polarized pump pulse induces non-stationary charge migration associated with an electronic wavepacket in benzene. The electron density (blue shaded area) migrates from one side of the molecule to the other with a period $\tau=504$ attoseconds. Black arrows correspond to the electronic flux density associated with this process (for $z=1 \mathrm{a}_{0}$ ).

In this work, we investigate charge migration in benzene induced by a linearly $x$-polarized pulse. A resonant pump pulse of $3.57 \mathrm{fs}$ duration (92 meV bandwidth) and $0.6 \mathrm{TW} / \mathrm{cm}^{2}$ intensity at $8.2 \mathrm{eV}$ photon energy is used to prepare a coherent electronic superposition of the $A_{1 g}$ ground state and a low-lying optically accessible $E_{1 u, x}$ electronic states [64]. The time period of the non-stationary charge migration corresponds to $\tau=504$ attoseconds (see Fig. 1). The timescale of the electronic motion of the wavepacket is much faster than the motion of nuclei [65, 66, which are kept frozen. The stateaveraged CASSCF $(6,6)$ method implemented in MOLPRO [67] is used with an aug-cc-pVTZ basis 68] to compute the singlet ground and low-lying electronic excited states of benzene, which is aligned in the $x y$-plane. As in Ref.[15], Multi-Reference Configuration Interaction with Single and Double excitations is employed to correct excitation energies.

To image charge migration and the associated fluxes in benzene, the time-resolved scattering signal is simulated using an expression for the differential scattering probability (DSP) of the form (in atomic units) [42, 52, 56]

$$
\frac{d P}{d \Omega}=\frac{d P_{e}}{d \Omega} \sum_{j}\left|\int d \mathbf{r}\left\langle\psi_{j}|\hat{n}(\mathbf{r})| \Phi(t)\right\rangle e^{-i \mathbf{Q} \cdot \mathbf{r}}\right|^{2},
$$

where $\frac{d P_{e}}{d \Omega}$ is the Thomson scattering cross section of a free electron, $\left|\psi_{j}\right\rangle$ is an eigenstate of the unperturbed Hamiltonian, $|\Phi(t)\rangle$ is an electronic wavepacket with $t$ as pump-probe time delay, $\hat{n}(\mathbf{r})$ is the electron density operator, and $\mathbf{Q}$ is the photon momentum transfer. In previous work, the numerical simulation of TRXS from the electronic wavepacket has been limited to atomic and simple molecular systems [42, 54, 58, 62, 69. For a general electronic wavepacket, the summation over $j$ in Eq. (1) runs over a complete set of eigenstates. Simulating scattering signals using a large number of eigenstates is usually not practical due to the associated computational cost. The scattering signal is shown to converge rapidly with respect to the number of eigenstates (see Fig. S2). All results reported in this work are computed using the 7 lowest-lying of eigenstates. All transition amplitudes of the density operator from the many-body eigenfunctions, i.e., $\left\langle\psi_{A_{1 g}}|\hat{n}(\mathbf{r})| \psi_{j}\right\rangle$ and $\left\langle\psi_{E_{1 u, x}}|\hat{n}(\mathbf{r})| \psi_{j}\right\rangle$, are simulated using the ORBKIT toolbox [0 72]. In the past, the summation over $j$ was restricted to the eigenstates spanning the wavepacket and the simulated scattering signals were used to understand the measured signals [45 48, 50]. Historically, it was believed that the DSP is proportional to the instantaneous electron density of the wavepacket. Neglecting the effect of electronic coherences was shown to be incorrect in similar contexts [42, 51, 52, 54, 58.

Here, we observe that the time-dependence of the momentum-space density also differs from that of the current flux density. The time-evolution of the signal obtained from Eq. (1) correlates with the time-derivative of the density calculated from first principles. The theoretical support for this correspondence is detailed in the Supporting Information (SI), where the time-evolution of the signals is derived for a general superposition state. To confirm these results numerically, we investigate the many-electron dynamics using the instantaneous variation of the one-electron density, $\partial_{t} \rho(\mathbf{r}, t)$, and the associate current flux density, $\mathbf{j}(\mathbf{r}, t)$. These are connected via the continuity equation, $\partial_{t} \rho(\mathbf{r}, t)=-\vec{\nabla} \cdot \mathbf{j}(\mathbf{r}, t)$. The one-electron density and the current flux density are computed from the time-dependent many-electron wavepacket, as described elsewhere [71, 72].

Time-resolved scattering patterns corresponding to an electronic wavepacket for different pump-probe delay times are presented in Fig. 2 a. The electronic wavepacket consists of a coherent superposition of two many-body electronic states which evolves according to

$$
\Phi\left(\mathbf{r}^{N}, t\right)=c_{A_{1 g}}(t) \psi_{A_{1 g}}\left(\mathbf{r}^{N}\right)+c_{E_{1 u, x}}(t) \psi_{E_{1 u, x}}\left(\mathbf{r}^{N}\right)
$$

The coefficients $c_{j}(t)=2^{-1 / 2} e^{-i \varepsilon_{j} t / \hbar}$ are associated with the ground state, $\psi_{A_{1 g}}\left(\mathbf{r}^{N}\right)$ at energy $\varepsilon_{A_{1 g}}$, and an optically accessible excited state, $\psi_{E_{1 u, x}}\left(\mathbf{r}^{N}\right)$ at energy $\varepsilon_{E_{1 u, x}}$. Eq. (1) is used to simulate the patterns shown in Fig. 2 a and presented in the $Q_{x}-Q_{y}$ plane $\left(Q_{z}=0\right)$. For representation purposes, the scattering pattern at $t=0$ 
(a)
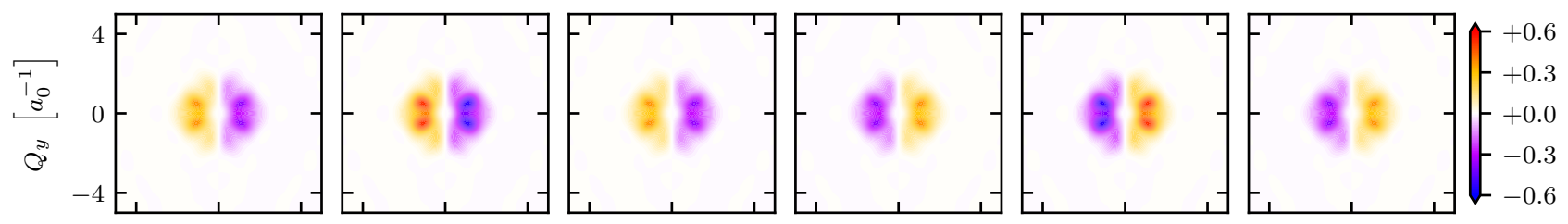

(b)
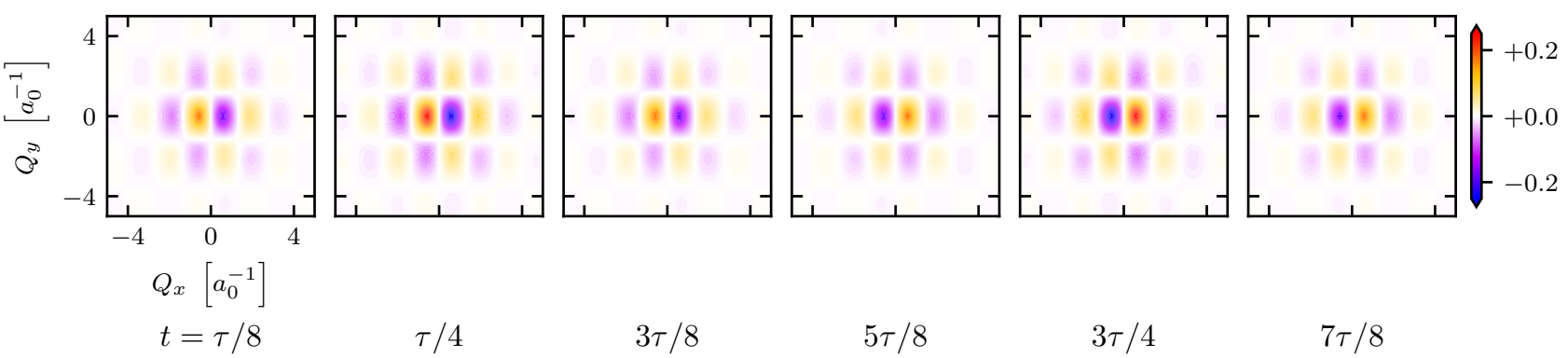

FIG. 2. Time-resolved signals in the $Q_{x}-Q_{y}$ plane $\left(Q_{z}=0\right)$ as a function of pump-probe delay times with a charge migration period of $\tau=504$ attoseconds for benzene. The time-resolved signal at time zero $t=0$ is subtracted from the signals at later delay times. (a) Scattering patterns obtained using Eq. (1), and (b) time-derivative of the momentum-space electron density $\rho(\mathbf{Q})$. The intensity of the scattering patterns are presented in units of $d P_{e} / d \Omega$.

is subtracted. The scattering patterns at $t=\tau / 4$ and $3 \tau / 4$ have opposite phase, whereas they are similar at $t=\tau / 8$ and $3 \tau / 8$, and at $t=5 \tau / 8$ and $7 \tau / 8$. Hence, the scattering patterns are sensitive to delay times, with a sin behaviour of period $\tau$. The time-derivative of the momentum space electron density, $\partial_{t} \rho(\mathbf{Q})$, is shown in the central panels of Fig. 2 $\mathrm{b}$. As visible from Figs. $2 \mathrm{a}$ and $2 \mathrm{~b}$, there is a one-to-one correspondence between the time-evolution of the scattering patterns obtained from Eq. (1) and the time-derivative of the electron density. Although the structure of $\partial_{t} \rho(\mathbf{Q})$ extends further in the $Q_{x}-Q_{y}$ plane, it contains the information of the DSP signal and the two quantities have the same period. As shown in the SI, the DSP signal is not exactly the time-derivative of the momentum-space electron density, but rather the convolution of its different contributions. This is the reason why the timescales correlate exactly with the dynamics but the spatial extent is different in both cases. According to Eq. (S12), the DSP signal from Eq. (1) also contains a contribution from the instantaneous density with $\sin ^{2}$ dependency of period $2 \tau$, which would lead to an asymmetry in the signal at $t=\tau / 4$ and $3 \tau / 4$. This asymmetry is not observed since the associated term is vanishingly small (see Fig. S1). Hence, the time-evolution of the experimental DSP signal yields quantitative information about the timescales involved in the time-derivative of the one-electron density. A more quantitative comparison can be obtained from Fig.3. which shows 1D cut of the DSP and the time-derivative of the momentum-space electron density. Despite differences at higher momenta, the pictures that emerges at low momenta are in good agreement. Low momenta are the most important, as they were shown to map the dynamics of valence electrons [16].

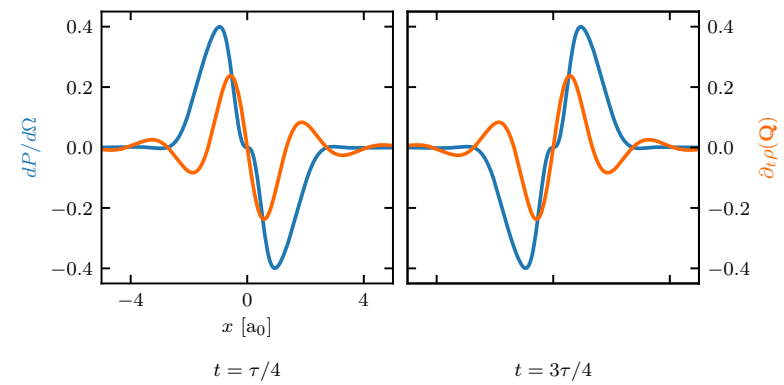

FIG. 3. Comparison of the time-resolved signals from Eq. (1) (blue) and the time-derivative of the momentum-space electron density $\rho(\mathbf{Q})$ (orange) along $Q_{x}\left(Q_{y}=Q_{z}=0\right)$ at pumpprobe delay times $t=\tau / 4$ and $t=3 \tau / 4$.

The time-dependent DSP signal encodes information about the time-evolution of the wavepacket in momentum-space. Hence, it contains information related to the velocity distributions. To reveal this information, we first reconstruct the current flux density from the many-electron wavepacket associated with the charge migration in benzene. The current flux density is a vector field in configuration space that maps the displacement of the volume elements of the one-electron density. Fig. 4 presents the time-derivative of the electron density (colour map) and the current flux density (arrows) at various delay times. These quantities are related via the electronic continuity equation, which describes the many-electron dynamics as the flow of a strongly correlated electronic fluid. The one-electron density is seen to migrate from left (violet/blue) to right (yellow/red) in the first half period of the charge migration process, before coming back. The nodal plane along the $y$-axis, which is a consequence of the pump pulse used to gener- 
ate this superposition state [64, is retained at all times.

The mechanistic information of the charge migration is encoded in the scattering patterns. However, it is not easy to know where are localised electrons that move in a certain region directly from the patterns. As can be seen from Fig. 4 a, the direction of the arrows correlates qualitatively with the time-resolved scattering patterns in momentum space depicted in the upper panels of the previous figure (see Fig. 2 a). The dominant electron flow is along the $x$-direction, with minor components in the $Q_{x}-Q_{y}$ plane at angles corresponding to the C-C bonds of the molecular scaffold. Both pictures are consistent and describe a bond-to-bond electron migration mechanism. On the other hand, the time-derivative of the oneelectron density reveals a more intricate nodal structure in the central panels of Fig. 2b. As discussed in previous work [71, 72, the derivative of the electronic density around the nuclei is sensitive to the choice of atomic basis set. The Fourier transform of the density reveals this sensitivity in momentum space.

The velocity field, calculated as $\mathbf{v}(\mathbf{r}, t)=\mathbf{j}(\mathbf{r}, t) / \rho(\mathbf{r}, t)$, offers an alternative representation of the charge migration mechanism. It is shown in Fig. 4p (arrows), along with the time-derivative of electron density (colour map). Although it contains mostly the same information as the current flux density, the velocity field is more easily related to the momentum observed in the DSP signal. The time-dependent rescaling through the one-electron density yields a better contrast of the electronic flow, which simplifies the direct comparison with scattering patterns. It can be observed that the electrons flow faster around the central carbon atoms, which contrasts with the picture offered by the current flux density. The latter predicts an homogeneous $\pi$-electron flow along the two C-C$\mathrm{C}$ units of the scaffold. The $\pi$-electron density is lower on the atoms than on the bonds. Rescaling the flux density by the density thus reveals an increased velocity at the central carbon atoms. This phenomenon is analogous to the Venturi effect in classical hydrodynamics, if we assimilate the reduction of the electron density to a reduction of the cross-section through which electrons flow. Since the volumetric flow rate is conserved, the smaller electron density implies an increased velocity and a reduced hydrodynamic pressure at the carbon atoms.

In conclusion, we have shown that ultrafast timeresolved X-ray scattering has potential to extract mechanistic information about the flow of electrons in a molecule out-of-equilibrium by mapping the electronic current flux density. The latter is related to the timevariation of the momentum-space density. The TRXS signal contains qualitative information about the instantaneous electronic velocity distribution and quantitative information about temporal electronic coherences. Cross-correlation with first-principle simulations can be used to reveal the electronic flux density, which contains the time- and space-resolved mechanistic details of the electron migration process. The experimental realization is limited by the time- and momentum-resolutions of TRXS. While benzene is beyond current experiments, the prediction remains valid for slower processes. This would require including nuclear motion in the theoretical treatment.

\section{ACKNOWLEDGEMENTS}

G.D. acknowledges for the Ramanujan fellowship (SB/S2/ RJN-152/2015). G.H. and V.P. are grateful for travel funding of the Freie Universität Berlin through the "Indo-German Partnership in Higher Education" program of the DAAD. J.C.T. , G.H., and V.P. are thankful to the Deutsche Forschungsgemeinschaft for funding through grant TR1109/2-1.

* gdixit@phy.iitb.ac.in

$\dagger$ jean-christophe.tremblay@univ-lorraine.fr

[1] F. Lépine, M. Y. Ivanov, and M. J. J. Vrakking, Nature Photonics 8, 195 (2014).

[2] S. R. Leone, C. W. McCurdy, J. Burgdörfer, L. S. Cederbaum, Z. Chang, N. Dudovich, J. Feist, C. H. Greene, M. Ivanov, R. Kienberger, U. Keller, M. F. Kling, Z. H. Loh, T. Pfeifer, A. N. Pfeiffer, R. Santra, K. Schafer, A. Stolow, U. Thumm, and M. J. J. Vrakking, Nature Photonics 8, 162 (2014).

[3] F. Remacle and R. D. Levine, Proc. Natl. Acad. Sci. U.S.A 103, 6793 (2006).

[4] A. D. Dutoi, M. Wormit, and L. S. Cederbaum, J. Chem. Phys. 134, 024303 (2011).

[5] J. J. Sakurai, Advanced quantum mechanics, Pearson Education India, 1967.

[6] I. Barth and J. Manz, Angew. Chem. Int. Ed. 45, 2962 (2006).

[7] I. Barth, J. Manz, Y. Shigeta, and K. Yagi, J. Am. Chem. Soc. 128, 7043 (2006).

[8] K. Nagashima and K. Takatsuka, J. Phys. Chem. A 113, 15240 (2009).

[9] K. Takatsuka and T. Yonehara, Phys. Chem. Chem. Phys. 13, 4987 (2011).

[10] M. Okuyama and K. Takatsuka, Bull. Chem. Soc. Jpn. 85, 217 (2012).

[11] D. J. Diestler, A. Kenfack, J. Manz, B. Paulus, J. F. Pérez-Torres, and V. Pohl, J. Phys. Chem. A 117, 8519 (2013).

[12] K. Takatsuka, T. Yonehara, K. Hanasaki, and Y. Arasaki, Chemical Theory beyond the BornOppenheimer Paradigm: Nonadiabatic Electronic and Nuclear Dynamics in Chemical Reactions, World Scientific, 2015.

[13] G. Hermann, B. Paulus, J. Pérez-Torres, and V. Pohl, Phys. Rev. A 89, 052504 (2014).

[14] K. Yamamoto and K. Takatsuka, Chem. Phys. Chem. 16, 2534 (2015).

[15] G. Hermann, C. M. Liu, J. Manz, B. Paulus, J. F. PrezTorres, V. Pohl, and J. C. Tremblay, J. Phys. Chem. A 
(a)
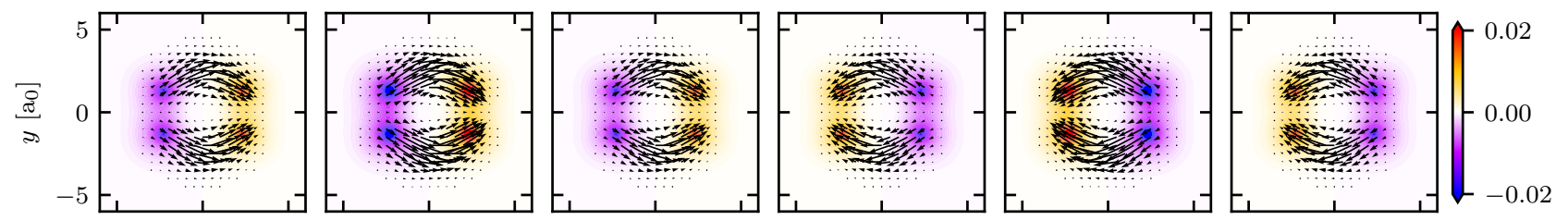

(b)
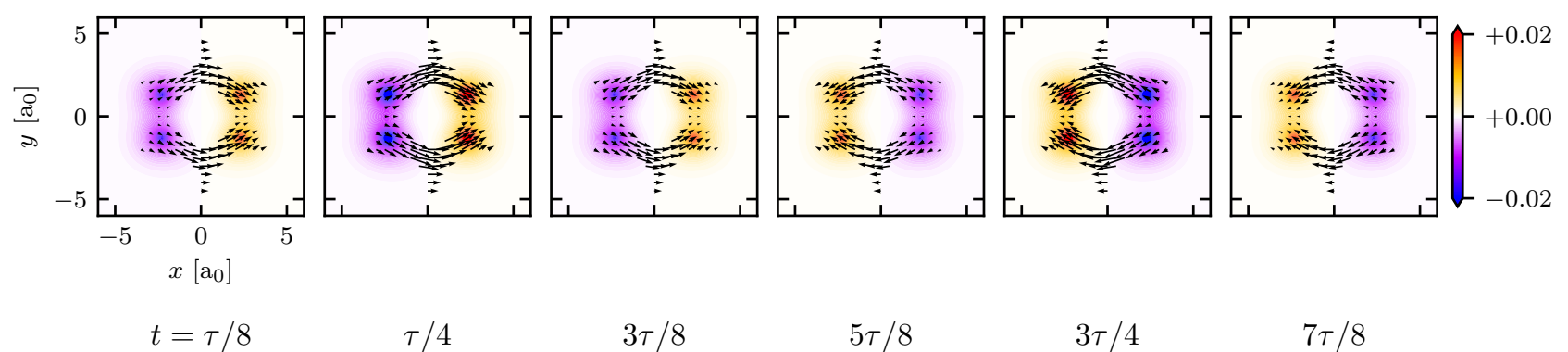

FIG. 4. Panel a: Fourier transform of the time-derivative of the momentum-space electron density $\rho(\mathbf{Q})$ (colour map) and the associated current flux density (arrows) as a function of pump-probe delay times. Panel b: Fourier transform of the time-derivative of the momentum-space electron density $\rho(\mathbf{Q})$ (colour map) and velocity field, $\mathbf{v}(\mathbf{r}, t)=\mathbf{j}(\mathbf{r}, t) / \rho(\mathbf{r}, t),(\operatorname{arrows})$ depicted only at positions where the electron density is above $\rho(r)>10^{-10} \mathrm{a}_{0}^{-3}$.

120, 5360 (2016).

[16] T. Bredtmann, M. Ivanov, and G. Dixit, Nature Communications 5, 5589 (2014).

[17] M. Okuyama and K. Takatsuka, Chem. Phys. Lett. 476, 109 (2009).

[18] D. Diestler, J. Phys. Chem. A 116, 2728 (2011).

[19] S. Patchkovskii, J. Chem. Phys. 137, 084109 (2012).

[20] I. Barth, J. Manz, Y. Shigeta, and K. Yagi, J. Am. Chem. Soc. 128, 7043 (2006).

[21] K. Takatsuka, Y. Arasaki, T. Yonehara, and K. Hanasaki, Chemical Theory Beyond the Born-Oppenheimer Paradigm: Nonadiabatic Electronic and Nuclear Dynamics in Chemical Reactions, World Scientific, 2015.

[22] V. Pohl and J. Tremblay, Phys. Rev. A 93, 012504 (2016).

[23] A. Schild, F. Agostini, and E. Gross, J. Phys. Chem. A 120, 3316 (2016).

[24] K. Renziehausen and I. Barth, Prog. Theor. Exp. Phys. 2018, 013A05 (2018).

[25] T. Schaupp, J. Albert, and V. Engel, Eur. Phys. J. B 91, 97 (2018).

[26] R. Matsuzaki and K. Takatsuka, J. Chem. Phys. 150, 014103 (2019).

[27] J. Als-Nielsen and D. McMorrow, Elements of modern $X$-ray physics, Wiley, New York, 2011.

[28] T. Ishikawa, H. Aoyagi, T. Asaka, Y. Asano, N. Azumi, T. Bizen, H. Ego, K. Fukami, T. Fukui, Y. Furukawa, S. Goto, H. Hanaki, T. Hara, T. Hasegawa, T. Hatsui, A. Higashiya, T. Hirono, N. Hosoda, M. Ishii, T. Inagaki, Y. Inubushi, T. Itoga, Y. Joti, M. Kago, T. Kameshima, H. Kimura, Y. Kirihara, A. Kiyomichi, T. Kobayashi, C. Kondo, T. Kudo, H. Maesaka, X. M. Marechal, S. Masuda, T.and Matsubara, T. Matsumoto, T. Matsushita, S. Matsui, M. Nagasono, N. Nariyama, H. Ohashi, T. Ohata, T. Ohshima, S. Ono, Y. Otake, C. Saji, T. Sakurai, T. Sato, K. Sawada, T. Seike, K. Shirasawa, T. Sugimoto, S. Suzuki, S. Takahashi, H. Takebe, K. Takeshita, K. Tamasaku, H. Tanaka, R. Tanaka, T. Tanaka, T. Togashi, K. Togawa, A. Tokuhisa,
H. Tomizawa, K. Tono, S. K. Wu, M. Yabashi, M. Yamaga, A. Yamashita, K. Yanagida, C. Zhang, T. Shintake, H. Kitamura, and N. Kumagai, Nature Photonics 6, 540 (2012).

[29] C. Pellegrini, A. Marinelli, and S. Reiche, Rev. Mod. Phys. 88, 015006 (2016).

[30] P. Emma, R. Akre, J. Arthur, R. Bionta, C. Bostedt, J. Bozek, A. Brachmann, P. Bucksbaum, R. Coffee, F. J. Decker, Y. Ding, D. Dowell, S. Edstrom, A. Fisher, J. Frisch, S. Gilevich, J. Hastings, G. Hays, P. Hering, Z. Huang, R. Iverson, H. Loos, M. Messerschmidt, A. Miahnahri, S. Moeller, H. D. Nuhn, G. Pile, D. Ratner, J. Rzepiela, D. Schultz, T. Smith, P. Stefan, H. Tompkins, J. Turner, J. Welch, W. White, J. Wu, G. Yocky, and J. Galayda, Nature Photonics 4, 641 (2010).

[31] T. Tanaka, Phys. Rev. Lett. 110, 084801 (2013).

[32] S. Kumar, J. Lee, M. S. Hur, and M. Chung, JOSA B 35, A75 (2018).

[33] S. Kumar, Y. W. Parc, A. S. Landsman, and D. E. Kim, Scientific Reports 6, 37700 (2016).

[34] C. H. Shim, Y. W. Parc, S. Kumar, I. S. Ko, and D. E. Kim, Scientific Reports 8, 7463 (2018).

[35] N. Hartmann et al., Nature Photonics 12, 215 (2018).

[36] J. Duris, S. Li, T. Driver, E. G. Champenois, J. P. MacArthur, A. A. Lutman, Z. Zhang, P. Rosenberger, J. W. Aldrich, R. Coffee, G. Coslovich, F.-J. Decker, J. M. Glownia, G. Hartmann, W. Helml, A. Kamalov, J. Knurr, J. Krzywinski, M.-F. Lin, M. Nantel, A. Natan, J. O'Neal, N. Shivaram, P. Walter, A. Wang, J. J. Welch, T. J. A. Wolf, J. Z. Xu, M. F. Kling, P. H. Bucksbaum, A. Zholents, Z. Huang, J. P. Cryan, and A. Marinelli, arXiv preprint arXiv:1906.10649 (2019).

[37] E. Lindroth, F. Calegari, L. Young, M. Harmand, N. Dudovich, N. Berrah, and O. Smirnova, Nature Reviews Physics 1, 107 (2019).

[38] L. Young et al., J. Phys. B 51, 032003 (2018).

[39] P. H. Bucksbaum, Science 317, 766 (2007).

[40] M. Peplow, Nature 544, 408 (2017).

[41] M. J. J. Vrakking, Physics 9, 112 (2016). 
[42] G. Dixit, O. Vendrell, and R. Santra, Proc. Natl. Acad. Sci. U.S.A. 109, 11636 (2012).

[43] M. J. J. Vrakking and T. Elsaesser, Nature Photonics 6, 645 (2012).

[44] J. Küpper, S. Stern, L. Holmegaard, F. Filsinger, A. Rouzée, A. Rudenko, P. Johnsson, A. V. Martin, M. Adolph, A. Aquila, S. Bajt, A. Barty, C. Bostedt, J. Bozek, C. Caleman, R. Coffee, N. Coppola, T. Delmas, S. Epp, B. Erk, L. Foucar, T. Gorkhover, L. Gumprecht, A. Hartmann, R. Hartmann, G. Hauser, P. Holl, A. Hömke, N. Kimme, F. Krasniqi, K. U. Kühnel, J. Maurer, M. Messerschmidt, R. Moshammer, C. Reich, B. Rudek, R. Santra, I. Schlichting, C. Schmidt, S. Schorb, J. Schulz, H. Soltau, J. C. Spence, D. Starodub, L. Strüder, J. Thgersen, M. J. J. Vrakking, G. Weidenspointner, T. A. White, C. Wunderer, G. Meijer, J. Ullrich, H. Stapelfeldt, D. Rolles, and H. N. Chapman, Phys. Rev. Lett. 112, 083002 (2014).

[45] J. M. Glownia, A. Natan, J. P. Cryan, R. Hartsock, M. Kozina, M. P. Minitti, S. Nelson, J. Robinson, T. Sato, T. van Driel, G. Welch, C. Weninger, D. Zhu, and P. H. Bucksbaum, Phys. Rev. Lett. 117, 153003 (2016).

[46] M. R. Ware, J. M. Glownia, N. Al-Sayyad, J. T. O'Neal, and P. H. Bucksbaum, arXiv preprint arXiv:1902.01972 (2019).

[47] M. P. Minitti, J. M. Budarz, A. Kirrander, J. S. Robinson, D. Ratner, T. J. Lane, D. Zhu, J. M. Glownia, M. Kozina, H. T. Lemke, M. Sikorski, Y. Feng, S. Nelson, K. Saita, B. Stankus, T. Northey, J. B. Hastings, and P. M. Weber, Phys. Rev. Lett. 114, 255501 (2015).

[48] M. P. Minitti et al., Faraday Discussions 171, 81 (2014).

[49] K. Pande, C. D. M. Hutchison, G. Groenhof, A. Aquila, J. S. Robinson, J. Tenboer, S. Basu, S. Boutet, D. P. DePonte, M. Liang, T. A. White, N. A. Zatsepin, O. Yefanov, D. Morozov, D. Oberthuer, C. Gati, G. Subramanian, D. James, Y. Zhao, J. Koralek, J. Brayshaw, C. Kupitz, C. Conrad, S. Roy-Chowdhury, J. D. Coe, M. Metz, P. L. Xavier, T. D. Grant, J. E. Koglin, G. Ketawala, R. Fromme, V. Srajer, R. Henning, J. C. H. Spence, A. Ourmazd, P. Schwander, U. Weierstall, M. Frank, P. Fromme, A. Barty, H. N. Chapman, K. Moffat, J. J. van Thor, and M. Schmidt, Science 352, 725 (2016).

[50] H. Yong et al., J. Phys. Chem. Lett. 9, 6556 (2018).

[51] J. Cao and K. R. Wilson, J. Phys. Chem. A 102, 9523 (1998).

[52] N. E. Henriksen and K. B. Moller, J. Phys. Chem. B 112, 558 (2008).

[53] U. Lorenz, K. B. Møller, and N. E. Henriksen, Phys. Rev. A 81, 023422 (2010).
[54] G. Dixit and R. Santra, J. Chem. Phys. 138, 134311 (2013).

[55] G. Dixit, J. M. Slowik, and R. Santra, Phys. Rev. Lett. 110, 137403 (2013).

[56] G. Dixit, J. M. Slowik, and R. Santra, Phys. Rev. A 89, 043409 (2014).

[57] R. Santra, G. Dixit, and J. M. Slowik, Phys. Rev. Lett. 113, 189301 (2014).

[58] K. Bennett, J. D. Biggs, Y. Zhang, K. E. Dorfman, and S. Mukamel, J. Chem. Phys. 140, 204311 (2014).

[59] G. Dixit and R. Santra, Phys. Rev. A 96, 053413 (2017).

[60] K. Bennett, M. Kowalewski, J. R. Rouxel, and S. Mukamel, Proc. Natl. Acad. Sci. U.S.A. 115, 6538 (2018).

[61] M. Kowalewski, K. Bennett, and S. Mukamel, Structural Dynamics 4, 054101 (2017).

[62] M. Simmermacher, N. E. Henriksen, K. B. Møller, A. M. Carrascosa, and A. Kirrander, Phys. Rev. Lett. 122, 073003 (2019).

[63] H.-C. Shao and A. F. Starace, Phys. Rev. A 99, 033413 (2019).

[64] D. Jia, J. Manz, B. Paulus, V. Pohl, J. C. Tremblay, and Y. Yang, Chem. Phys. 482, 146 (2017).

[65] H. Mineo, S. Lin, and Y. Fujimura, Chem.l Phys. 442, 103 (2014).

[66] V. Despré, A. Marciniak, V. Loriot, M. Galbraith, A. Rouzée, M. Vrakking, F. Lépine, and A. Kuleff, J. Phys. Chem. Lett. 6, 426 (2015).

[67] H. J. Werner, P. J. Knowles, G. Knizia, F. R. Manby, M. Schütz, P. Celani, T. Korona, R. Lindh, A. Mitrushenkov, G. Rauhut, K. R. Shamasundar, T. B. Adler, R. D. Amos, A. Bernhardsson, A. Berning, D. L. Cooper, M. J. O. Deegan, A. J. Dobbyn, F. Eckert, E. Goll, C. Hampel, A. Hesselmann, G. Hetzer, T. Hrenar, G. Jansen, C. Koppl, Y. Liu, A. W. Lloyd, R. A. Mata, A. J. May, S. J. McNicholas, W. Meyer, M. E. Mura, A. Nicklass, D. P. O'Neill, P. Palmieri, D. Peng, K. Pflueger, R. Pitzer, M. Reiher, T. Shiozaki, H. Stoll, A. J. Stone, R. Tarroni, T. Thorsteinsson, and M. Wang, See http://www molpro. net (2012).

[68] T. H. Dunning Jr, J. Chem. Phys. 90, 1007 (1989).

[69] M. Simmermacher, N. E. Henriksen, and K. B. Møller, Phys. Chem. Chem. Phys. 19, 19740 (2017).

[70] G. Hermann, V. Pohl, J. C. Tremblay, B. Paulus, H. C. Hege, and A. Schild, J. Comp. Chem. 37, 1511 (2016).

[71] V. Pohl, G. Hermann, and J. C. Tremblay, J. Comput. Chem. 38, 1515 (2017).

[72] G. Hermann, V. Pohl, and J. C. Tremblay, J. Comput. Chem. 38, 2378 (2017). 